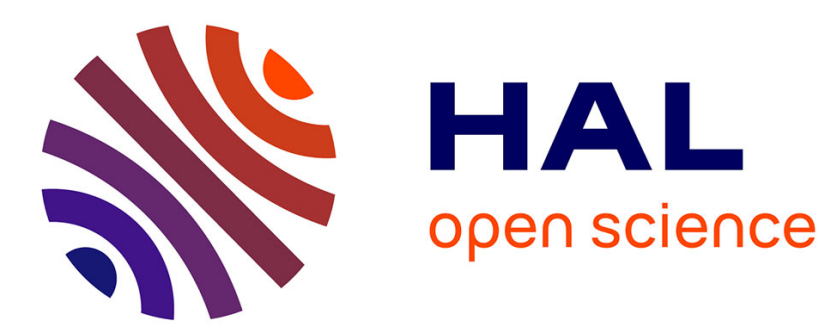

\title{
Modelling of the chemical potential in precipitation models
}

\author{
Joel Lepinoux
}

\section{To cite this version:}

Joel Lepinoux. Modelling of the chemical potential in precipitation models. Philosophical Magazine, 2021. hal-03363131

\section{HAL Id: hal-03363131 \\ https://hal.science/hal-03363131}

Submitted on 3 Oct 2021

HAL is a multi-disciplinary open access archive for the deposit and dissemination of scientific research documents, whether they are published or not. The documents may come from teaching and research institutions in France or abroad, or from public or private research centers.
L'archive ouverte pluridisciplinaire HAL, est destinée au dépôt et à la diffusion de documents scientifiques de niveau recherche, publiés ou non, émanant des établissements d'enseignement et de recherche français ou étrangers, des laboratoires publics ou privés. 


\title{
Modelling of the chemical potential in precipitation models
}

\author{
J. Lépinoux
}

\section{ARTICLE HISTORY}

Compiled 12th March 2021

Univ. Grenoble Alpes, CNRS, Grenoble INP, SIMaP, F-38000 Grenoble, France

Corresponding author: joel.lepinoux@grenoble-inp.fr

\begin{abstract}
Modelling of precipitation in concentrated binary alloys requires to revisit the fundamentals of precipitation. While no experimental approach can provide information accurate enough for this purpose, Atomic Kinetic Monte Carlo simulations (AKMC) can generate large amounts of data produced in well-controlled conditions.

Recently, the extensive analysis of such data has revealed new features about the evolution of clusters during precipitation. In particular, it has been shown that their free energy evolves linearly with the chemical potential of monomers $\mu_{1}$. Their volume is another complex function which evolves with $\mu_{1}$. This quantity can be easily extracted from AKMC simulations: its variable part depends on $C_{1}$, the concentration of monomers and $M_{1}$, the concentration of pure matrix sites, neglected in classical approaches.

Cluster dynamics (CD) behaves as a bridge between atomistic simulations and phenomenological models. CD can be applied to concentrated alloys provided one can describe the evolution of the free energy and the volume of clusters as functions of $\mu_{1}$. Therefore, modelling of $M_{1}$ is the last obstacle to build a complete CD scheme, no longer limited by the concentration or the supersaturation of alloys.

For this purpose, two methods are examined and to predict the evolution of $M_{1}$ during precipitation and compared with the results of AKMC simulations. One is derived from a generalisation of the exact solution in $1 \mathrm{D}$ while the other extrapolates the results of AKMC simulations.
\end{abstract}

Keywords: AlLi alloys, precipitation, computer simulation, clusters, Monte Carlo

\section{X-scale modelling of precipitation}

The so-called KWN framework (e.g. [1]) derived from the work of Wagner and Kampmann [2] is a powerful numerical means to model precipitation in various industrial situations [3, 4]. The strength of this framework is to rely on simple phenomenological laws to describe nucleation, growth and coarsening. Some of the parameters necessary to set up these laws can be obtained from thermodynamic databases and others can be identified through dedicated experiments [5, 6]. Nevertheless, concerning the case of concentrated alloys or alloys of moderate concentrations but of high supersaturation, the validity of these phenomenological laws is at least questionable.

Improving these laws requires first a deeper understanding of precipitation in concentrated alloys. For this purpose, the best candidates are certainly not modern industrial alloys with many components, very anisotropic and incoherent precipitates or with a complex architecture. To optimise our chances to bring out new features specific to concentrated alloys, it is much better to consider binary alloys as simple as possible. Similarly, while modern experimental techniques are essential for the study of real alloys, for our concern, numerical simulations and in particular AKMC simulations is the only means to rise the reliable information we need. However, to validate or to update the required classical phenomenological laws, it is necessary to complete AKMC simulations with another numerical tool: Cluster Dynamics (CD).

Indeed, CD is an alternative method closely related to the Classical Nucleation Theory (CNT) [7, 8]. $\mathrm{CD}$ is based on mesoscopic parameters, the so-called condensation and emission coefficients $\left(\beta_{n}\right.$ and $\alpha_{n}$ ) which can be defined consistently with the atomic potential used to perform AKMC simulations or 
with quantities which can be directly extracted from such simulations [9]. In that sense, it is a valuable link between atomistic simulations and phenomenological approaches. It is also an invaluable tool to check the validity of our understanding of precipitation, while it is absolutely not adapted to treat complex industrial situations.

In section 2 we briefly recall our choice for a model alloy and the four cases we consider as in our previous works, e.g. [10]. In addition we provide some information about computing time. Section 3 is devoted to the ingredients necessary to succeed the transition between AKMC simulations and CD calculations. We recall how to define $\mu_{1}$, the chemical potential of monomers, which allows for the managing of free energy and volume of clusters during precipitation. Then we recall the notion of exclusion volume, to be used in section 4 , devoted to the modelling of $M_{1}$, the key factor to define $\mu_{1}$. In section 4 , first we briefly recall the previous attempts to account for the exclusion effect. Then we propose two ways to model $M_{1}$. Both have advantages and drawbacks and are likely to complement each other to better secure the managing of $\mu_{1}$.

\section{The model material and the simulation method}

\subsection{A model alloy}

The high solubility limit of $\delta \mathrm{Al}_{3} \mathrm{Li}$ clusters in aluminium makes this ordered alloy of $\mathrm{L}_{2}$ structure a good candidate to examine precipitation in concentrated alloys. In addition, precipitates are coherent with the aluminium matrix, thus using AKMC simulations on a rigid lattice (see [11] for details about this technique) is fully justified. For this purpose we have developed a model of atomic interactions limited to first and second nearest-neighbours (NN1 and NN2), based on the parametrisation proposed by Garland and Sanchez [12] for the two effective pair interaction parameters. All details about building this classical pair potential can be found in [9].

Clusters have been studied in the temperature range $50^{\circ} \mathrm{C}-300^{\circ} \mathrm{C}$ but as in previous works, we focus on two cases at $85^{\circ} \mathrm{C}$ and two cases at $200^{\circ} \mathrm{C}$; the solubility limits are respectively $2.7 \%$ and $6.3 \%$ (all mentioned concentrations are atomic concentrations). For each temperature we have selected one case exhibiting the well-known stages of nucleation, growth and coarsening and another one of higher supersaturation for which this distinction is no longer possible. These four cases are: (1) $85^{\circ} \mathrm{C}$ and $C_{0}=4.75 \%$, (2) $200^{\circ} \mathrm{C}$ and $C_{0}=8.25 \%,(3) 200^{\circ} \mathrm{C}$ and $C_{0}=10 \%$ and (4) $85^{\circ} \mathrm{C}$ and $C_{0}=9 \%$. We have checked a posteriori that only monomers are mobile, thus this alloy is also a good choice for comparison between future CD calculations and present AKMC simulations. AKMC simulations performed in this work are based on a vacancy diffusion model [11] and the exchange between the vacancy and one of its $12 \mathrm{NN} 1$ is solved by a residence time algorithm [13].

\subsection{AKMC simulations}

Contrary to a simple thermodynamic Monte Carlo model (i.e. based on elementary exchanges between close atoms), this method cannot be easily parallelised. Therefore, the computing time is still controlled by the power of single processors as it was usual for most problems a few decades ago. Figure 1 shows typical examples of the required CPU time as a function of the excess of chemical potential of monomers $\Delta \mu$ (cf. Section 2.2). As can be seen, it is possible to run these simulations until this quantity reduces by one order of magnitude from its initial value, starting from a random distribution. Two simulation box sizes have been used: $200^{3}$ atoms (the green curve in Figure 1) and $500^{3}$ atoms (other curves). Comparisons of simulations performed in the same conditions with these two box sizes have shown that from the beginning of quasi-equilibrium (indicated by a green disk in Figure 1) the CPU time is proportional to the size of the simulation box. Thus, in Figure 1, the CPU time corresponding to the green curve have been multiplied by $(500 / 200)^{3}=15.625$ to compare with other curves, which explains the large dispersion of data when $\Delta \mu$ vanishes.

The advantage of a large box is the quality of output information which remains fairly good up to very small values of $\Delta \mu$ while the advantage of a small box is the possibility to get acceptable information in a reasonable amount of CPU time. With a large box, the green curve in Figure 1 would have required about two years of CPU instead of 1.5 month.

Whatever the precipitation framework, the alloy is implicitly assumed to be close to equilibrium. 
In dilute alloys, this condition is straightforward and CD can be launched from a random state. But in concentrated alloys this is no longer possible because cluster properties are no longer constant during precipitation. To manage the evolution of cluster properties (free energy and volume) during precipitation, the calculations have to be launched from a realistic and well-documented initial state of quasi equilibrium (QE), which includes the right properties of clusters [10].

If the considered alloy is a good candidate for atomistic simulations on a rigid lattice, then the required initial conditions can be easily obtained from AKMC simulations, performed at least up to the onset of QE (for an introduction about this notion, see [14]). But in practice it might be a good idea to run simulations up to smaller values of $\Delta \mu$. In a previous work we have shown that this is mandatory to provide better predictions of the evolution of cluster volumes [15]. For similar reasons, it will be shown in the last section that it is also required to better manage $M_{1}$. This is why it is interesting to run in parallel two simulations, one using a large box to get accurate information on early stages and the other one using a small box to get more approximate results but over a much larger time range.

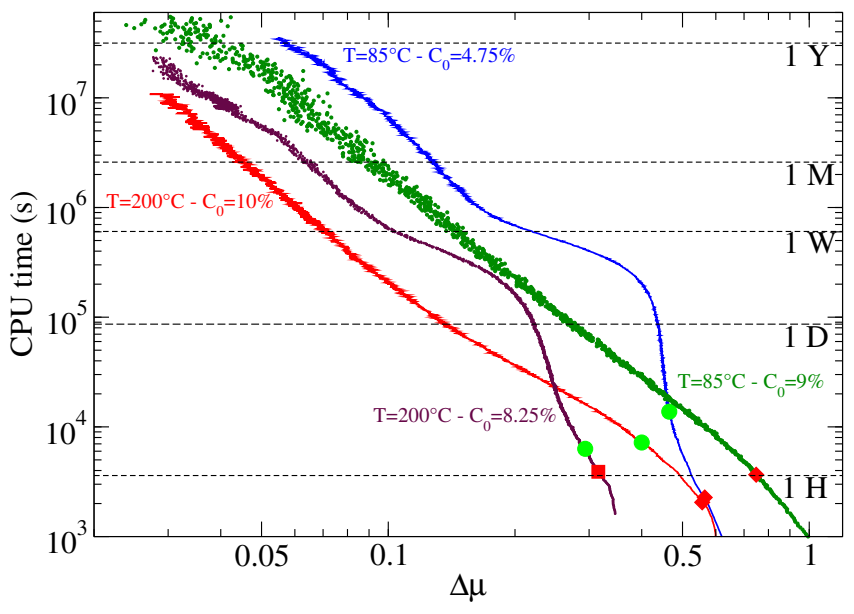

Figure 1.: CPU time taken to reach $\Delta \mu$ (the excess of chemical potential of monomers - cf. section 3.1) for the four main cases. Dashed lines show CPU time after 1 hour, 1 day, 1 week, 1 month and 1 year (performed on Intel@ Xeon E-2186M CPU 2.90GHz processors). When visible, green and red disks (colour online) indicate the beginning of quasi-equilibrium and that of nucleation, respectively.

\section{From AKMC simulations to Cluster Dynamics}

\subsection{The chemical potential of monomers}

Classically, precipitation kinetics is reported versus the physical time, a choice which seems fully justified. Nevertheless, to understand the evolution of cluster properties, the most convenient parameter is not the physical time but the chemical potential of monomers $\mu_{1}$. Contrary to the global or average chemical potential, $\mu_{1}$ can be simply defined from a few fundamental quantities which are easy to measure in AKMC simulations [14]. In practice, we use the normalised form:

$$
\bar{\mu}_{1}=\bar{h}_{1}+\ln \left(\frac{C_{1}}{M_{1}}\right)
$$

where the notation $\bar{x}$ denotes the quantity x normalised by $k_{B} T$ (the Boltzmann constant times the temperature); $h_{1}$ is the monomer enthalpy, $C_{1}$ is the concentration of monomers and $M_{1}$ that of pure matrix sites. Here, a pure matrix site is an aluminium site whose all NN1 and NN2 are aluminium atoms (i.e. matrix). If its nature is changed from $\mathrm{Al}$ to $\mathrm{Li}$ it becomes a monomer and vice versa. Setting $\bar{\mu}_{1}=\bar{\mu}_{1}^{S L}$ at the solubility limit, to simplify we define $\triangle \mu=\bar{\mu}_{1}-\bar{\mu}_{1}^{S L}$ as the excess of chemical potential of monomers (a positive quantity, while $\bar{\mu}_{1}$ and $\bar{\mu}_{1}^{S L}$ are negative). As can be seen in Figure 2, during a 
precipitation kinetics at constant temperature, $\triangle \mu$ converges toward zero in the long-time limit. The initial value of $\triangle \mu$ is a function of the supersaturation and the temperature.

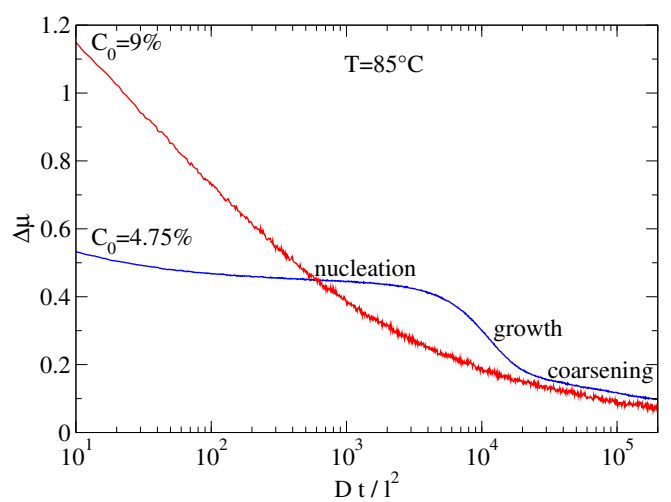

(a)

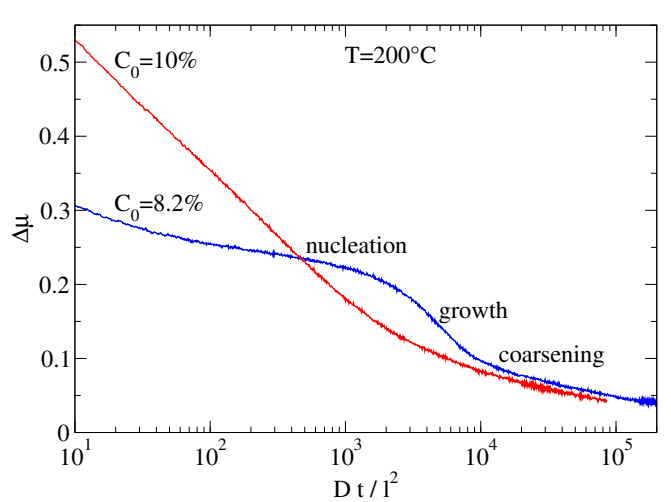

(b)

Figure 2.: The excess of chemical potential of monomers $\Delta \mu$ vs. the physical time multiplied by the diffusion coefficient D over $l^{2}(l$ is the lattice parameter) for 2 concentrations and 2 temperatures. Three well-defined stages are clearly distinguished for the low concentrations (blue curves online) while this distinction vanishes for the high concentrations (red curves online) (from [15]).

Two types of behaviour are highlighted in Figure 2, reporting the evolution of $\triangle \mu$ vs. the normalised physical time. Note that the initial values of $\triangle \mu$ for the two cases at $85^{\circ} \mathrm{C}$ are about twice larger than for the cases at $200^{\circ} \mathrm{C}$. Although these two Figures seem almost identical, there is no simple way to superimpose two similar curves (i.e. the two red or the two blue curves).

\subsection{The required quantities to set up CD}

Predicting the evolution of a cluster distribution with CD consists in solving a set of differential Equations:

$$
\dot{C}_{n}=\left(\alpha_{n+1} C_{n+1}+\beta_{n-1} C_{n-1}\right)-\left(\alpha_{n}+\beta_{n}\right) C_{n}
$$

for $n>1$, where $n$ is the size (the number of solute atoms) of clusters in concentration $C_{n}$. The absorption coefficient $\beta_{n}$ is a kinetic coefficient related to $D$, the diffusion coefficient, $R_{n}$, the cluster radius and $C_{1}$, the monomer concentration:

$$
\beta_{n}=4 \pi D R_{n} C_{1} / l^{3}
$$

Here we assume that only monomers are mobile. This simple expression is based on the long distance diffusion, which is very likely to be inappropriate in the case of high concentration. Several corrections can be found in the literature to account for superimposition of diffusion fluxes; for a review, see [8]. Comparing CD with AKMC results will tell us if these corrections are valid or not. In the present work we focus on the thermodynamic state of the alloy during precipitation. While the kinetic is controlled by the $\beta_{n}$ coefficients, the thermodynamic is controlled by the ratios $\left(\beta_{n} / \alpha_{n+1}\right)$ :

$$
\left(\frac{\beta_{n}}{\alpha_{n+1}}\right)=\left(\frac{C_{1}}{M_{1}}\right)\left(\frac{P_{n \rightarrow n+1}^{*}}{P_{n+1 \rightarrow n}^{*}}\right)
$$

Remark that $\left(C_{1} / M_{1}\right)$ is the variable component of $\mu_{1}$ while the constant component is hidden inside the ratio $\left(P_{n \rightarrow n+1}^{*} / P_{n+1 \rightarrow n}^{*}\right)$, related to $F_{n}^{*}$, the free energy of $n$-mers: 


$$
\left(\frac{P_{n \rightarrow n+1}^{*}}{P_{n+1 \rightarrow n}^{*}}\right)=\exp \left(\frac{h_{1}}{k_{B} T}\right) \exp \left(-\frac{F_{n+1}^{*}-F_{n}^{*}}{k_{B} T}\right)
$$

The ratio $\left(P_{n \rightarrow n+1}^{*} / P_{n+1 \rightarrow n}^{*}\right)$ can be easily measured from the analysis of clusters build in AKMC simulations [9]. The symbol "*" indicates that the values taken by these quantities account for the possibility of coagulation with other clusters around. In other words, one considers a given system in a given state. This is why these coefficients are so difficult to predict with a sufficient accuracy without the help of AKMC simulations [16]. However, provided that the system is in QE, the ratio $\left(P_{n \rightarrow n+1}^{*} / P_{n+1 \rightarrow n}^{*}\right)$ can be easily fitted as a function of $n$ [10]. Then, knowing that the asymptotic state of the solid solution is equivalent to its state at the solubility limit, and that $F_{n}^{*}$ varies linearly with $\triangle \mu$ between these two points, the last missing information to apply Equation 8 during the whole precipitation kinetics is $M_{1}$.

\subsection{Notion of exclusion volume}

In classical models the volume of a cluster is simply taken proportional to the number of solute atoms it contains [8]. This amounts to consider that a cluster is equivalent to a sample of the bulk precipitate phase of the same size. This solution is acceptable for very large precipitates but does not make sense for very small clusters and generally speaking, it is questionable for cluster sizes involved in nucleation and growth stages.

Another point of view consists in considering the 'exclusion volume' which is composed of the solute skeleton and of all NN1 and NN2 of these solute atoms (for the most recent work about this notion see [15]; section 4.2 comes back to this notion). By exclusion we mean that the available acceptor sites of a cluster cannot capture a solute atom without increasing its size by one unit. For convenience, we prefer to use the number of excluded sites $N_{e x}(n)$ instead of the associated exclusion volume $V_{e x}(n)$. The monomer is the simplest case: it contains the solute atom, its $6 \mathrm{NN} 2$ and its $12 \mathrm{NN} 1$, thus $N_{e x}(1)=19$. To further simplify, we consider the normalised exclusion volume $X(n)=N_{e x}(n) / n$.

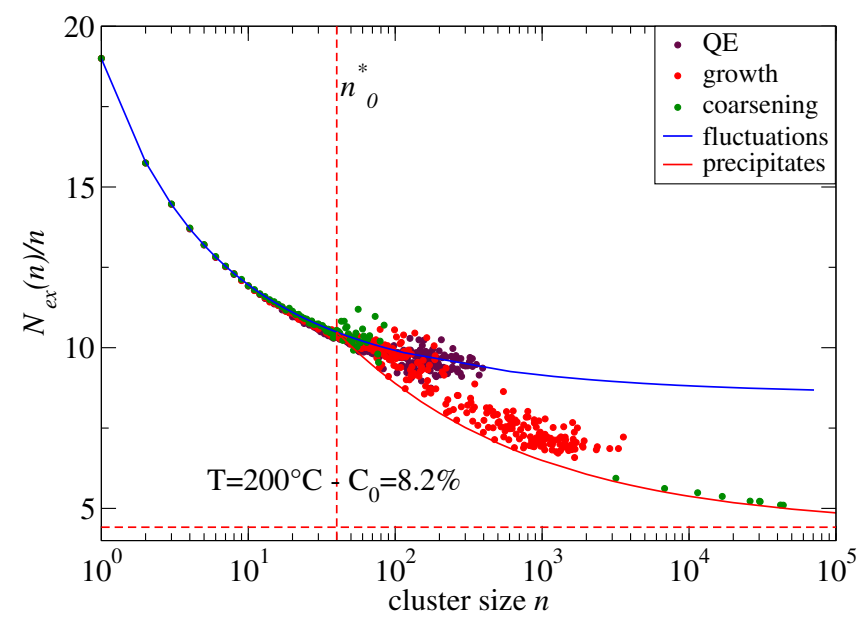

Figure 3.: Evolution of the normalised number of excluded sites vs. the cluster size, during a precipitation kinetics in a case which exhibits the three classical stages of precipitation (cf. Figure 2). $n_{0}^{*}$ is the minimum of the critical size. The upper curve (blue online) is related to fluctuations at the solubility limit. The lower curve (red online) is the asymptote for precipitates and the horizontal dashed line is related to the bulk precipitate phase (i.e. 4.415 at $\mathrm{T}=200^{\circ} \mathrm{C}$ ) (from [15]).

Figure 3 shows a typical example for a moderate supersaturation and a high concentration. Two domains appear, diverging for a value $n_{0}^{*}$ which corresponds approximately to the minimum value reached by the critical size for nucleation at the very beginning of nucleation [15]. In the first approximation, the branch related to the fluctuations (left side) of the solid solution can be considered as constant during precipitation. On the contrary, for a given precipitate size (right side), $X(n)$ decreases from 
an initial value and converges toward an asymptotic value. As for the free energy, if one knows $X(n)$ around the onset of $\mathrm{QE}$, the modelling of the evolution of $X(n)$ during precipitation is fairly easy [15]. Note that the exclusion volume is only the starting point to get the cluster radius which enters for instance in Equation (3). The procedure to derive $R_{n}$ from this exclusion volume is discussed in section 4.2 which describes one possible way to evaluate $M_{1}$.

\section{Modelling of $M_{1}$}

\subsection{First attempts to account for the exclusion effect}

Although the notion of exclusion or matrix frustration is fairly recent, Frenkel's cluster gas model [17] is to our knowledge the very first attempt to account for a possible effect of the solute concentration on the expression of equilibrium concentrations $\widetilde{C}_{n}$ :

$$
\left(\frac{\widetilde{C}_{n}}{1-\sum_{k} \widetilde{C}_{k}}\right)=\left(\frac{\widetilde{C}_{1}}{1-\sum_{k} \widetilde{C}_{k}}\right)^{n} \exp \left(-\frac{F_{n}-n F_{1}}{k_{B} T}\right)
$$

The corrective terms $\left(1-\sum_{k} \widetilde{C}_{k}\right)$ were obtained assuming that each cluster occupies only one atomic site, independently of its size. Of course, this correction was found negligible for dilute alloys and to our knowledge it has never been really used.

In 2006, a more elaborated cluster gas model was proposed to remove the main approximations of the original model [18]. As 50 years earlier, the goal was to obtain a better expression of equilibrium concentrations $\widetilde{C}_{n}$. Our current definition of the exclusion volume or equivalently the number of excluded sites (see section 3.3) arises from this work. $N_{k, n}$, the number of sites excluded by $k$-mers to a $n$-mer, was obtained considering spherical clusters:

$$
N_{k, n}=4\left(\frac{4 \pi}{3}\right)\left(\frac{R_{k}+R_{n}}{l}\right)^{3}
$$

where the pre-factor 4 comes from the number of atoms per unit cell and $l$ is the lattice parameter; $R_{k}$ and $R_{n}$ are the respective radii of clusters $k$ and $\mathrm{n}$. These radii were derived from the number of excluded sites by a monomer, denoted $N_{e x}(1)$ in the previous section but $N_{1,1}$ in the notation of Equation (7). Thus, as $N_{1,1}=19$, we obtain $\left(R_{1} / l\right)=0.5214$. The same work introduced also the exclusion factors $M_{n}$ to replace the Frenkel's corrective terms in Equation (6):

$$
M_{n}=\prod_{k}\left(1-C_{k}\right)^{N_{k, n}}
$$

Finally, the proposed solution was:

$$
\left(\frac{\widetilde{C}_{n}}{M_{n}}\right)=\left(\frac{\widetilde{C}_{1}}{M_{1}}\right)^{n} \exp \left(-\frac{F_{n}-n F_{1}}{k_{B} T}\right)
$$

Combined with free energy calculations (assuming no interaction between clusters), for the first time it was possible to accurately reproduce cluster distributions produced by AKMC simulations of moderately concentrated AlZr/AlSc alloys without extra corrections [18]. However, a few years later, further works put into evidence the limits of this model at higher solute concentrations. Although the global form of Equation (9), was correct, the route proposed to calculate the exclusion factors $M_{n}$ was reproducing a historical mistake. Indeed, clusters properties, e.g. free energy or volume, were still considered as 'intrinsic' properties, and the effect of exclusion by other clusters was taken as a simple additive effect. It has been extensively explained in previous works, e.g. [16, 14], why this simple 
picture, inherited from studies of dilute alloys, is no more than a good approximation for moderate concentrations.

\subsection{With help of the exact solution in $1 D$}

In 2010, starting from the exact solution of this problem in 1D proposed by Yilmaz and Zimmermann [19], Berthier et al. [20] have shown that the above model could not be applied to the 1D case, although as in 3D, it gives acceptable results for moderately concentrated systems. The authors suggested a generalised solution for 2-3D problems. However, the differences between the very special 1D case and the 2-3D cases are so numerous and severe that the existence of an exact solution in 2-3D is still an assumption. Furthermore, to our knowledge, the proposed solution has never been applied and compared with AKMC simulations in $2 \mathrm{D}$ or $3 \mathrm{D}$.

The direct calculation of $M_{1}$ can be easily added to the outputs of AKMC simulations or performed separately [14], thus comparing any model with AKMC simulations is now fairly easy. Moreover, the present CD framework, i.e. Equation (9), has a big advantage over the previous 2006 model [18]: only $M_{1}$ is required. And as $M_{1}$ is a very special case among all possible $M_{n}$, working around this generalized solution has appeared as a starting point worthy to consider for our current needs. For the special case $n=1$, the generalised solution (Equation (13) in [20]) writes:

$$
M_{1}=(1-\theta) \prod_{n}\left(1-\frac{C_{n}}{(1-\theta)}\right)^{\widetilde{N}_{e x}(n)}
$$

The differences with Equation (8) concern the factor $(1-\theta)$ which appears twice and the definition of $\widetilde{N}_{e x}(n)$, slightly different from that of $N_{k, 1}$ given by Equation (7). But these two differences are coupled. Concerning $\widetilde{N}_{e x}(n)$, Berthier et al. [20] assumed that a cluster (approximated by a sphere) is composed of a 'hard' exclusion core $N_{\text {core }}(n)$ and a 'soft' exclusion shell. Here, 'soft' and 'hard' refer to whether it is possible to share atomic sites between several clusters (shell) or not (core), a key distinction which was neglected in [18]. Thus, whatever the definition of $N_{\text {core }}(n), \widetilde{N}_{e x}(n)$ writes:

$$
\widetilde{N}_{e x}(n)=N_{e x}(n)-N_{\text {core }}(n)
$$

Coming back to the original work of Yilmaz and Zimmermann [19] one can give two possible interpretations of $\theta$ which cannot be distinguished in the 1D case:

$$
\theta=\sum_{n} C_{n} N_{\text {core }}(n)
$$

i.e. the total sum of all $N_{\text {core }}$ whether clusters are precipitates or fluctuations of the solid solution, and

$$
\theta=N_{p} \sum_{n} n C_{n}
$$

which is equivalent to a sample of bulk precipitate phase containing all solute atoms of the system. Equation (13) can be seen as the long-time limit of Equation (12). This ambiguity suggests that the generalisation of the 1D case should be considered with great care and also with some flexibility.

The first pre-product factor in Equation (10) is supposed to represent the probability for a monomer to be located outside the total volume of hard exclusion. Thus, a priori, here $\theta$ should be given by Equation (12). The second occurrence of $\theta$ as a normalizing term is more ambiguous but for the sake of consistency the solution should be the same as in the first occurrence. Again it accounts for the fact that the core of clusters is a volume of strict exclusion by contrast with its shell which can be partly shared with other clusters. As there is no strong argument to eliminate the other interpretation of $\theta$, 
we have tested the four possible combinations to see which one provides the best approximation of the measured values of $M_{1}$.

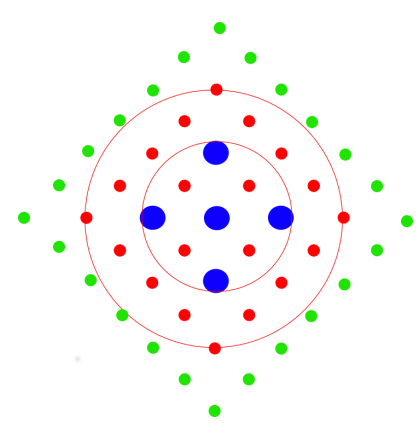

Figure 4.: Notions of exclusion and core volumes of clusters. A 2D example of 5-mer. Large disks (blue online): solute atoms, defining a first circle. Small disks inside the larger circle (red online): matrix atoms surrounding the cluster and linked to at least one solute atom by at least one NN1 or one NN2 bond, defining the largest circle, i.e. the exclusion volume. Other disks (green online): sites surrounding the exclusion volume of the cluster, not linked to any solute site of the cluster. Note that all NN1 bonds and all NN2 bonds of atoms located inside the first zone (blue disks) are linked with atoms which all belongs to the exclusion volume (blue or red disks). In the shell zone between the two circles, the sites can be shared between several clusters. Consequently, they contribute to the core volume of the cluster with a weight $0<w<1$ while all blue sites contribute with a weight $w=1$.

For this purpose, we need to define the quantity $N_{\text {core }}(n)$ introduced in Equation (11). After various trials, we have selected a solution based on the exclusion volume of a monomer. The principle consists in calculating a weight coefficient for each atom of a monomer, the solute atom itself and its 18 neighbours (NN1 or NN2), defined as the proportion of bonds between the considered atom and other atoms of the cluster (cf. Figure 4). Of course the solute atom itself counts for 1: by construction, all its 12 NN1 and its $6 \mathrm{NN} 2$ are atoms of the cluster. Any of these $12 \mathrm{NN} 1$ has 9 bonds with the cluster and any of these $6 \mathrm{NN} 2$ has 5 bonds with the cluster. Therefore, after averaging, the sum of all coefficients gives: $1+(6 \mathrm{X} 5+12 \mathrm{X} 9) / 18=8.666$ (sites).

Then, we can calculate the radii associated with $N_{\text {core }}(1)=8.666$ and $N_{e x}(1)=19$. This gives $R_{e x}(1)=1.043 l, R_{\text {core }}(1)=0.803 l$ and $\Delta_{\text {shell }}=R_{e x}(1)-R_{\text {core }}(1)=\varepsilon l$ with $\varepsilon=0.24$. To calculate $R_{\text {core }}(n)$ we assume that $\Delta_{\text {shell }}$ is a characteristic of the exclusion of monomers (the smallest possible probe to explore the surface of a cluster), independently of the size of the targeted clusters. Therefore, we postulate that $R_{\text {core }}(n)$ can be defined as:

$$
R_{\text {core }}=R_{e x}(n)-\varepsilon l
$$

Accounting for this expression into Equation (11) leads to:

$$
N_{\text {core }}(n)=\left[\frac{16 \pi}{3}\right]\left(\left(N_{\text {ex }}(n) /\left[\frac{16 \pi}{3}\right]\right)^{1 / 3}-\epsilon\right)^{3}
$$

or more simply: 


$$
N_{\text {core }}(n)=\left(N_{e x}^{1 / 3}(n)-\varphi\right)^{3}
$$

with $\varphi=19^{(1 / 3)}-(26 / 3)^{(1 / 3)}=0.614$ and $\varepsilon=\varphi /(16 \pi / 3)^{(1 / 3)}$.

With this definition of $N_{\text {core }}(n)$, the best combination for the first and the second occurrences of $\theta$ in Equation (10) is Equation (12) and Equation (13), respectively. Other combinations give excellent results in some cases and large deviations in other cases, thus they have been eliminated.

Finally, the complete expression proposed for $M_{1}$ writes:

$$
M_{1}=\left(1-\sum_{n} C_{n} N_{\text {core }}(n)\right) \prod_{n}\left(1-\frac{C_{n}}{\left[1-N_{p} \sum_{k} k C_{k}\right]}\right)^{N_{e x}(n)-N_{\text {core }}(n)}
$$

Note that the quantity between bracket is constant during the whole precipitation kinetics. Of course $N_{\text {core }}(n)$ can be defined following the method used to define $N_{\text {core }}(1)$ and similarly, it can be extracted from AKMC simulations instead of using Equations (16-17). The measured values slowly diverge from that predicted by Equation (16) but their ratio can be easily fitted. Nevertheless, the comparison of these two ways to define $N_{\text {core }}(n)$ has showed that Equations (16-17) lead to better results than the measured values of $N_{\text {core }}(n)$, which tends to validate a posteriori the assumption invoked to derive Equation (14).

In Figure 5, for the four cases considered in this paper, we have reported the ratio of the predicted values and the measured values of $M_{1}$ as a function of the measured values of $\Delta \mu$. The curves start on the right side of the figure; the first point is taken around the minimum of $M_{1}$.

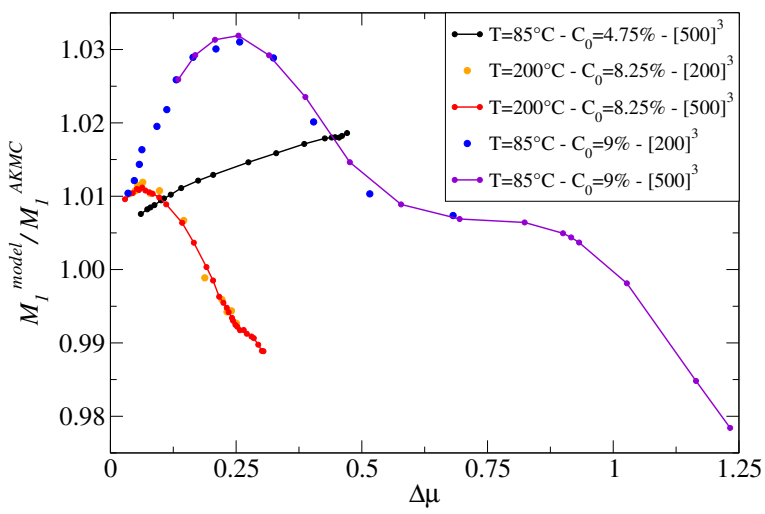

Figure 5.: The ratio $M_{1}$ (predicted) $/ M_{1}$ (measured) vs. $\triangle \mu$ (measured) for cases 1-3. Two sizes of the simulation box have been compared: $[200]^{3}$ and $[500]^{3}$. The curves start at the minimum of $M_{1}$.

In case 1, the relative error starts around $2 \%$ and ends under 1\%, thus Equation (17) gives a good approximation. The case 2 exhibits a different behaviour, but the relative error is even lower than in case 1 , although it also ends around $1 \%$. Note that a change of size of the simulation box from [200] ${ }^{3}$ to $[500]^{3}$ sites does not change the result.

As expected, the cases 3 and 4 exhibit distortions related to the existence of large cluster colonies (see [15]), mostly during the growth stage. However, the consequences of these distortions remain at an acceptable level for case 3 as can be seen in Figure 5. On the contrary, for case 4 (not reported here) a large deviation appears at the very beginning of nucleation and the relative error reaches almost $20 \%$ before quickly decreasing during the coarsening stage, with a relative error of the same magnitude as in case 2 . 
By construction, this model considers well-defined clusters, thus if coagulation is too strong, one cannot expect relevant results, which does not lower its interest for other cases.

Note that in the four cases studied here, these curves seem to end with a residual relative error of about $1 \%$. Thus, we have performed the same analysis at the solubility limits for $85^{\circ} \mathrm{C}$ and $200^{\circ} \mathrm{C}$ : again we have obtained a relative error of $1 \%$. Thus, for some reason, it seems that Equation (17) gives values which are systematically $1 \%$ too high. Therefore, we propose to account for this correction, so that all curves end with a negligible error in the long-term limit, which is more satisfying.

Note also that the present definition of $N_{\text {core }}(n)$ is slightly different from that used in the exact solution in $1 \mathrm{D}$. Indeed, in $1 \mathrm{D}, N_{\text {core }}(n)=n[19,20]$, while the present model would give $N_{\text {core }}(n)=$ $n+1$.

Despite these limitations, Equation (17) provides a valuable approximation, but only comparisons with other structures of precipitates and matrix could tell us if this model can be generalised.

\subsection{A direct method}

This method follows the principle already used to predict the evolution of cluster free energy or cluster volume during precipitation (cf. Section 2). It is also a generalisation of the work initiated in [14] with an oversimplified interaction potential. This method is based on two requirements:

(i) the end of the curve is known from the AKMC study of the system at the solubility limit (SL)

(ii) the beginning of the curve (at least up to the onset of QE) is known from AKMC simulations.

First we have to predict the asymptotic value of $M_{1}$ in a supersaturated system knowing its value at the solubility limit. To simplify we assume that in the asymptotic state the system is composed of two phases separated by a planar interface as previously done in [14]. The parameters are:

- $C_{0}$, the total solute concentration

- $C_{S L}$, the solubility limit $\left(2.7 \%\right.$ at $85^{\circ} \mathrm{C}$ and $6.3 \%$ at $\left.200^{\circ} \mathrm{C}\right)$

- $C_{P}$, the solute concentration in the bulk precipitate $\left(1 / 4.135\right.$ at $85^{\circ} \mathrm{C}$ and $1 / 4.415$ at $\left.200^{\circ} \mathrm{C}\right)$

With these notations, the asymptotic value of $M_{1}$ writes:

$$
M_{1}^{\infty}=M_{1}^{S L}\left(\frac{C_{p}-C_{0}}{C_{p}-C_{S L}}\right)
$$

As can be seen in Figure 6 these calculated asymptotic values are in excellent agreement with the evolution of $M_{1}$ vs. $\Delta \mu$ despite the small size of our simulation boxes.

Starting from a pure random distribution, $M_{1}$ first decreases, reaches a minimum and increases up to the asymptotic value. Whether the system is at equilibrium or not does not play any role on the measurements of $M_{1}$ or that of $C_{1}$. However, to manage $M_{1}$ as a function of $\Delta \mu$ one should be keep in mind that Equation (1) implicitly assumes equilibrium or in practice quasi-equilibrium. Thus, between the minimum of $M_{1}$ and the onset of QE (indicated on each curve by a blue disk), the evolution of $M_{1}$ vs. $\Delta \mu$ should be considered with great care. Note that for the four cases considered here, the minimum of $M_{1}$ is close to the onset of QE, except in Figure 6c. In this case, the part of the curve before the onset of QE is remarkably consistent with the rest of the curve. Consequently, in this case, it can be efficiently used to adjust the fit as a function of $\Delta \mu$, but a priori, one should be very careful with such situations.

As soon as the information extracted from AKMC simulations is rich enough to build accurate predictions of the free energy [10] and the volume of clusters [15], one has to perform tests to evaluate the predictability of $M_{1}$ and decide if one needs more information or if AKMC simulations can be stopped. Note that as with the previous method, using a $[200]^{3}$ simulation box is sufficient to get reliable information. To fit $M_{1}$, we have used a polynomial function which by construction ends at the asymptotic values calculated above for $\Delta \mu=0$; the number of required terms (order 3 or 4 ) is adjusted on the curvature of the curves. As an exercise, in Figure 6, we have tried to find the minimum learning range required to predict the whole curve from the onset of QE: it shows that it is necessary to run AKMC beyond the onset of QE indicated in Figure 6 by green disks (superimposed with the blue disk in Figure 6c).

The two less supersaturated cases exhibit a slight change of curvature during the growth stage which cannot be predicted with this method. Nevertheless, the maximum relative error is lower than $0.5 \%$ in 
Figure $6 \mathrm{a}$ and less than $1 \%$ in Figure $6 \mathrm{~b}$. The maximum relative error in the two higher supersaturated cases is negligible.

Note that this initial prediction of the whole curve is not mandatory. A better prediction can be obtained if one applies this method step by step but this requires more calculations as well as an elaborated adaptative algorithm to optimise the fit, depending on results and various constraints (to be tested within the CD framework).

It is recalled that in classical models, $M_{1}$ is implicitly taken equal to 1 . As can be seen in Figure 6 the error can be rather large, especially in the first stages of precipitation.

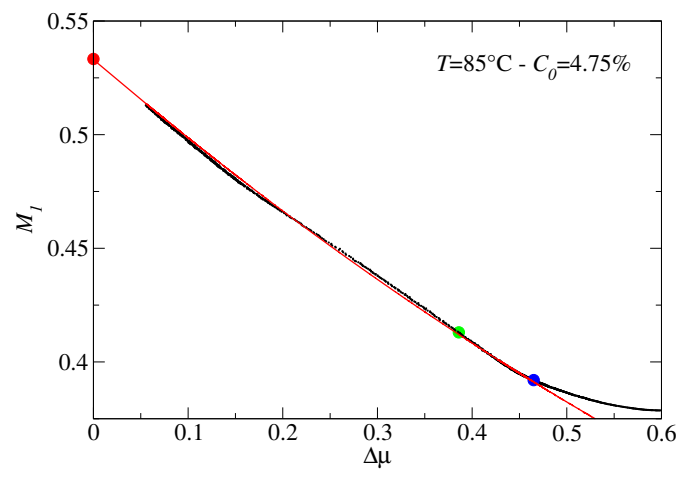

(a)

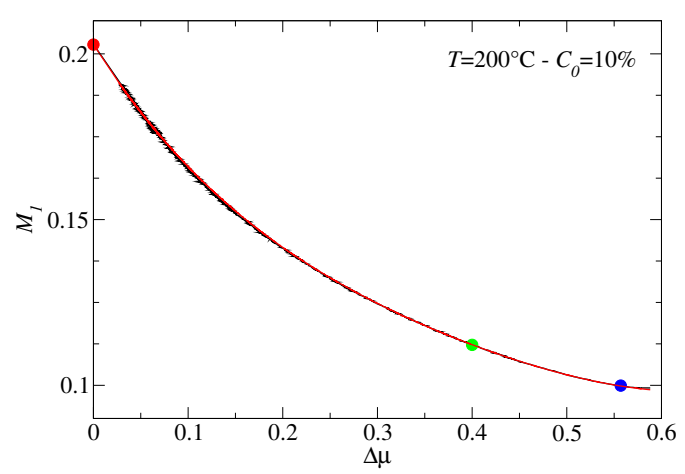

(c)

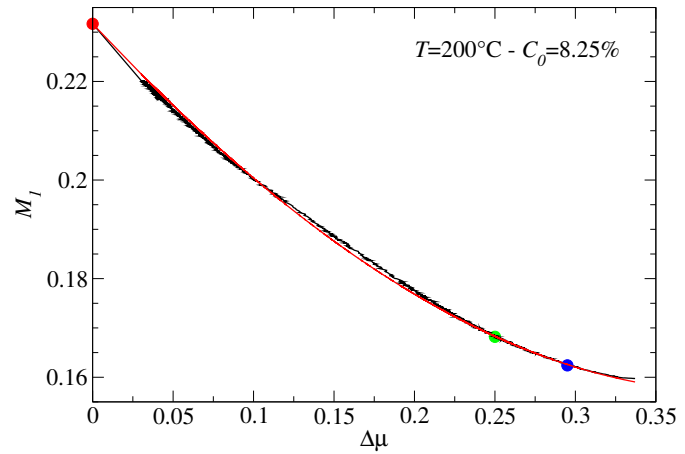

(b)

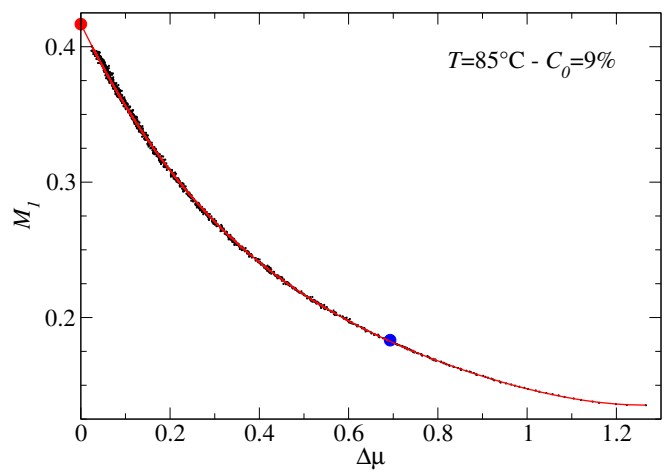

(d)

Figure 6.: Fitting and extrapolating $M_{1}$ values extracted from AKMC simulations (black curve online). The curves start at the minimum of $M_{1}$, on the right side of the figures. To predict a complete curve, the learning domain has to extend up to the second symbol (green online). Note that in case (d), this symbol is superimposed with that indicating the onset of QE (blue online). The last symbol (red online) corresponds to the asymptote described by Equation (18).

\section{Conclusion}

Accurate calculations of $M_{1}$ and then $\Delta \mu$ require to have access to the coordinates of all solute atoms. This is possible only in atomistic simulations, but such simulations are limited by the size of systems one can consider and by the computing time. Consequently, the use of a mesoscopic model like CD or a phenomenological model like the KWN formalism remains essential.

These two approaches have in common the fact that they cannot access to the real space. The needs of these 2 techniques are different in nature but at this point we focus on that of CD. $M_{1}$ must be modelled as finely as possible because it is explicitly included into the emission coefficients and also allows accounting for the evolution of free energy and volume of clusters during precipitation.

As already suggested in [14], the method using information obtained from AKMC simulations run at least up to the onset of quasi equilibrium, already used to predict the evolution of free energy and exclusion volume as functions of $\Delta \mu$, is very efficient to predict $M_{1}$. It can be used either step by step, 
but in this case $M_{1}$ must be regularly evaluated, or to predict $M_{1}$ up to its asymptotic value, but this requires to run AKMC simulations beyond the onset of QE. These two ways have different computing costs and the most convenient one depends on the imposed conditions.

The semi-analytical method derived from the exact solution in 1D provides results whose quality depends on conditions. By construction, it is not appropriate if the coagulation of clusters plays an important role. The comparison with values extracted from AKMC simulations run up to the onset of QE is sufficient to estimate if the method is trustable or not for those conditions. When it is, it can be used only step by step but the required calculations are simple and the increase in computing time is negligible. As this method uses the exclusion volumes and concentrations of all cluster classes, it might be also interesting to check a posteriori the consistency of results obtained with the method based on AKMC simulations. This method is also likely to be very useful to study precipitation in case of complex thermal paths, but this will require extensive tests.

Even precipitation in dilute alloys could take advantage of these results after some simplification (the case 1 is a good starting point). The next step is to gather all these ingredients to build an enhanced CD framework. The main point to investigate will be the role of coagulation in the highest supersaturated cases, to find the best way to deal with these cluster colonies while preserving the reliability and the simplicity of this method. Concerning phenomenological models, an alternative to generalised laws to describe the three stages might be to explicitly manage $M_{1}$ as in CD. For this purpose, a coupling with $\mathrm{CD}$ to manage the solid solution, in particular $C_{1}$, could be a way to consider.

\section{Acknowledgements}

Dr. E. Clouet is gratefully acknowledged for providing his AKMC package. Dr C. Sigli is warmly acknowledged for fruitful discussions.

\section{References}

[1] M. Perez, M. Dumont and D. Acevedo, Implementation of classical nucleation and growth theories for precipitation, Acta Materialia 56 (2008) pp. 2119-2132

[2] R. Wagner and R. Kampmann, Homogeneous second phase precipitation, in Materials Science and Technology, Eds. R. W. Cahn, P. Haasen, and E. J. Kramer, Vol. .5, Chapt. 4, Verlag Chemie GmbH, Weinheim (1991) pp. 213 - 304.

[3] M. Nicolas and A. Deschamps, Characterisation and modelling of precipitate evolution in an Al$Z n-M g$ alloy during non-isothermal heat treatments, Acta Materialia 51 (2003) pp. 6077-6094.

[4] M. Perez and A. Deschamps, Microscopic modelling of simultaneous two-phase precipitation: application to carbide precipitation in low-carbon steels, Mater Sci Eng A 360 (2003) pp. 214-219.

[5] A. Deschamps, C. Sigli, T. Mourey, F. de Geuser, W. Lefebvre and B. Davo, Experimental and modelling assessment of precipitation kinetics in an Al-Li-Mg alloy, Acta Materialia 60 (2012) pp. $1917-1928$

[6] A. Deschamps and M. Perez, Mesoscopic modelling of precipitation: A tool for extracting physical parameters of phase transformations in metallic alloys, C. R. Physique 11 (2010) pp. 236-244.

[7] G. Martin, Reconciling the Classical Nucleation Theory and Atomic Scale Observations and Modeling, Advanced Engineering Materials 8(12) (2006) pp. 1231-1236.

[8] E. Clouet, Modeling of nucleation processes, in Fundamentals of Modelling for Metals Processing, D.U. Furrer and S.L. Semiatin (Eds.), Materials Park, OH, ASM Handbook, vol. 22A Fundamentals of modelling for metals processing (2010) pp.203-219.

[9] J. Lépinoux and C. Sigli, Extracting free energy of clusters in concentrated binary alloys from atomistic Monte Carlo simulations, Modelling. Simul. Mater. Sci. Eng. 27 (2019) p. 08500.

[10] J. Lépinoux, Evolution of clusters free energy during precipitation in concentrated binary alloys, Phil. Mag. Letters, 100(8) (2020) pp. 402-411.

[11] F.Soisson and G. Martin, Monte Carlo simulations of the decomposition of metastable solid solutions: Transient and steady-state nucleation kinetics, Phys. Rev. B 62(1) (2000) pp. 203-214.

[12] J.S. Garland, J.M. Sanchez, Cluster variation method calculation of the metastable aluminiumlithium phase diagram, in Kinetics of Ordering Transformations in Metals, H. Chenand V.K. 
Vasudevan (Eds.), TMS, Warrendale, PA (1992) pp. 207-216.

[13] A. Clouet, M. Nastar and C. Sigli, Nucleation of $\mathrm{Al}_{3} \mathrm{Zr}$ and $\mathrm{Al}_{3} \mathrm{Sc}$ in aluminium: from kinetic Monte Carlo simulations to classical theory, Phys Rev. B 69 (2004) pp. 064109-(1-14).

[14] J. Lépinoux and C. Sigli, Multiscale modelling of precipitation in concentrated alloys: from atomistic Monte Carlo simulations to cluster dynamics: I thermodynamics, Phil. Mag. 98(1) (2017) pp. $1-19$.

[15] J. Lépinoux, What can we learn from the exclusion volume of fluctuations and precipitates?, to be published in Phil. Mag. (2021). https://doi.org/10.1080/14786435.2021.1882710

[16] J. Lepinoux and C. Sigli, On the effect of concentrated solid solutions on properties of clusters in a model binary alloy, Phil. Mag. 96(10) (2016) pp. 955-971.

[17] J. Frenkel, Kinetic Theory of Liquids, Dover Publications, New York (1955).

[18] J. Lépinoux, Contribution of matrix frustration to the free energy of cluster distributions in binary alloys, Phil. Mag. 86 (2006) pp. 5053-5082.

[19] M.B. Yilmaz and F.M. Zimmermann, Exact cluster size distribution in the one-dimensional Ising model, Phys Review E 71 (2005) p. 026127

[20] F. Berthier, I. Braems, E. Maras, J. Creuze and B. Legrand, Extending cluster dynamics to concentrated and disordered alloys: The linear-chain case, Acta Materialia 58(7) (2010) pp.2387-2398. 